\title{
Application of Sensor Technology for the Efficient Positioningand Assembling of Ship Blocks
}

\author{
Sangdon Lee ${ }^{1}$, SeongbaeEun ${ }^{2}$, Jai Jin Jung ${ }^{3}$, Hacheol Song ${ }^{4}$ \\ ${ }^{1}$ Department of Multimedia Engineering, Mokpo National University, Muan-Kun, Korea \\ ${ }^{2}$ Department of Information Communication Engineering, Hannam University, Daejon, Korea \\ ${ }^{3}$ Department of Multimedia Engineering, Dankook University, Chunan, Korea \\ ${ }^{4}$ Department of Naval Architecture and Marine Engineering, Mokpo National University, Muan-Kun, Korea
}

\begin{abstract}
This paper proposes the application of sensor technology to assemble ship blocks efficiently. A sensor-based monitoring system is designed and implemented to improve shipbuilding productivity by reducing the labor cost for the adjustment of adequate positioning between ship blocks during pre-erection or erection stage. For the real-time remote monitoring of relative distances between two ship blocks, sensor nodes are applied to measure the distances between corresponding target points on the blocks. Highly precise positioning data can be transferred to a monitoring server via wireless network, and analyzed to support the decision making which needs to determine the next construction process; further adjustment or seam welding between the ship blocks. The developed system is expected to put to practical use, and increase the productivity during ship blocks assembly.
\end{abstract}

KEY WORDS: Sensor technology; Remote monitoring; Ship block; Block positioning; Pre-erection/Erection stage.

\section{INTRODUCTION}

Block construction method has been generalized to the most of worldwide modern shipyards since 1950's because of higher dock turnover rate compared to the previous methods in the shipbuilding history. This method includes assembling ship structural elements into larger hull blocks, and finally, constructing a whole ship structure.

In the process of ship blocks assembly, two (or more) target ship blocks need to be assembled together within allowable margin of manufacturing accuracy. If the corresponding ship blocks are out of manufacturing accuracy, trimmed parts of blocks have to be adjusted and lacking parts supplemented by welding. These extra processes which come from insufficient manufacturing accuracy result in the increase of shipbuilding cost during pre-erection and in-dock erection stage (Han, 2006).

In mid- or small-sized shipyards, block positioning and assembling processes are accomplished by the experience of shipyard workers. In order to secure assembly precision, they have used very primitive tools like tape measures, lines, weights and their manual observation, etc.

Corresponding author: Sangdon Lee

E-mail:sdlee@mokpo.ac.kr
Nowadays, a laser-optical device becomes popular for the measurement and correction of ship block deformation. Even though the laser-optical device can be used in ship block assembly processes, continual manual operation needs to monitor whether the target blocks are in position or not. And this device has more restrictions for the practical use of ship block assembly as explained below;

(1) This is applicable only when an observer can see the target on ship block because the light from the device to be reflected to measure the distance and the angle from the base point.

(2) When a ship block is moved close to the other, the target point to be measured can be hidden.

Those restrictions explain that an observer needs to change his position very frequently in order to use a laseroptical device as a tool of ship block assembly.

In this paper, a remote monitoring system developed will be introduced to put to practical use for ship block assembly. This system consists of sensor nodes, wireless communication and a monitoring server. The monitored data from sensor nodes about relative distances between the ship blocks being assembled together are transferred to a monitoring server through wireless communication, and analyzed to support the decision making which needs to determine the next construction process; further adjustment or seam welding between the ship blocks. 


\section{RELATED WORKS}

Accurate manufacturing and precise assembly of unit blocks are essential to improve the productivity in preerection and erection stage. Thus, precision management is one of the crucial factors affecting quality control and reliability of a ship structure. Shipyards consistently have been putting many efforts for the development of better precision management in shipbuilding process, and providing allowable limit of deformation control for typical ship hull blocks. Han et al.(2006) analyzed major sources of errors in the process of ship block assembly. It suggested criteria for precision management for mid-sized unit block and evaluated the precision of block assembly.

Location systems applying sensor technology has focused on using triangulation(Hightower and Borriello, 2001a-c) to estimate distance between two points using direction or strength of the received signal, or difference of the arrival time of signal, etc. Some developed systems to estimate locations include Active Bagde (Want et al., 1992) from AT\&T, Cricket (Priyantha et al., 2000) from MIT and RADAR (Bahl and Padmanabhan, 2000) from Microsoft. Other approaches (Gutmann et al., 1998; Fox et al., 1999; Nicolescu and Nath, 2001) have tried to determine relative position of a sensor node using RFID and UWB (Ultra Wide Band) or wireless network. However estimation errors of the previous studies were up to several meters. It goes beyond the limit of quality control for ship construction. During typical ship block assembly, only a-few millimeters error is allowed.

Sensor technology has been applied to many shipbuilding applications. Examples include the real time monitoring of location of parts or smaller blocks, and block movement until it is properly positioned. The RFID and the wireless LAN technology(Kang et al., 2008) were used as a part of monitoring system. Lee (2009) proposed to use sensor technology directly to measure the precision of block assembly. The sensor-based monitoring system described in this paper exploits sensor technology to measure and monitor the process of ship block assembly more precisely for practical use in shipyards.

\section{MONITORING SHIP BLOCK ASSEMBLY}

For monitoring ship block assembly, several pairs of corresponding points have to be determined on the faces of targeted blocks to be assembled. Accordingly, a set of sensor nodes are capable of measuring relative distance between corresponding points on each target block. Figure 1 shows the overall concept of the real-time ship block assembly monitoring system. Fig. 1(a) shows that 4-pairs of corresponding points were determined to monitor the relative distances between two ship blocks being assembled. A sensor node and target board is attached at each point.

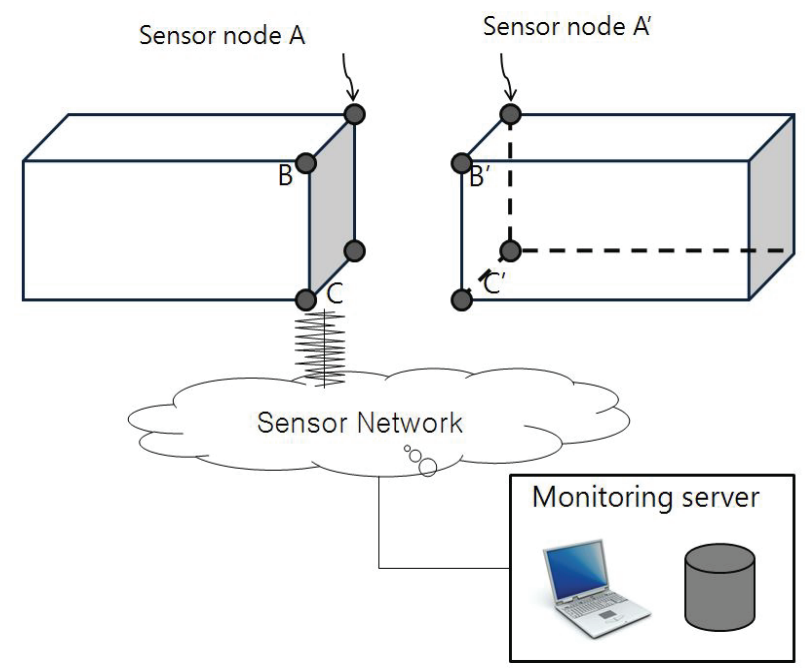

(a) overall configuration

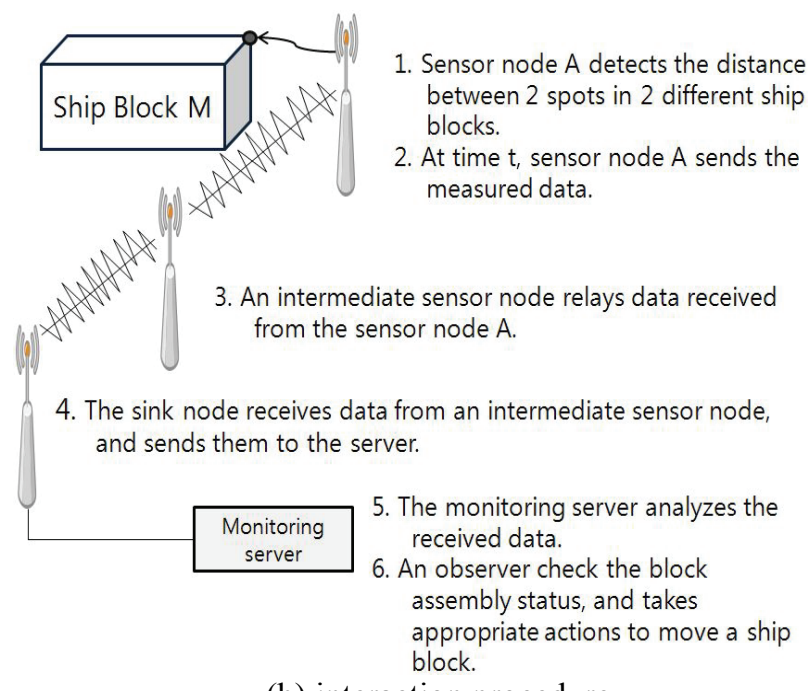

(b) interaction procedure

Fig. 1 Concept of monitoring ship block assembly.

During ship block assembly process, relative distance for each corresponding points is transmitted to a remote monitoring server via wireless relay devices. Fig. 1(b) shows an interaction procedure about data transmission between system components. Based on the real-time information about the status of block assembly, an observer determines appropriate actions for controlling movement of the ship blocks being assembled.

\section{DESIGN OF SENSOR-BASED MONITORING FOR SHIP BLOCK ASSEMBLY}

For monitoring of ship block assembly, two types of information needs to be gathered for each pair of corresponding points; one is the distance between two points, and the other is the declination; the position gap between the real-time position and target position for block assembly. 


\section{Measuring distances between two blocks}

In order to control the process of block assembly, it is essential to get information about the distance between ship blocks. Although previous sensor-based location systems have used various approaches to measure distance between two objects, those were not applicable to the practical ship block assembly application because of low precision. By contrast shipbuilding application allows only up to severalmillimeters measurement error, the previous traditional sensor-based applications up to several meters. In order to enhance the precision of distance measurement using sensor, Lee (2009) has tried to use more sophisticated embedded sensor nodes which include ultrasonic sensor devices to measure distance between two points. However the performance of an ultrasonic device is apt to be sensitive to environmental factors such as temperature, humidity, etc. This tendency became more intensified if the two blocks get close. In order to improve this drawback we combine two different distance measuring methods; the ultrasonic and a linear position transducer approach. If the measuring distance between two pointsis farther than $20 \mathrm{~cm}$ ultrasonic approach is used, and nearer than $20 \mathrm{~cm}$, a linear position transducer is used to get fine-grained distance within several-millimeters precision.

\section{Correcting positional declination}

During the process of assembling two ship blocks, one block is considered as fixed, and the other block is moved towards the fixed block. To get the right position of the block for assembly, it needs to be moved in 3 dimensional spaces. When we regard the block's sliding direction as $\mathrm{X}$-axis, a block also has to be moved in direction of $Y$ and $Z$-axis. Fig. 2 shows an example of the required positional declination. In this figure, the distance between a pair points $\mathrm{P} 1$ and $\mathrm{P} 2$ is measured. Let's denote the 2-D plane where P1, P2 is residing as S1, S2 respectively. Let's assume that two points on a plane S2 is $\mathrm{P} 2$ and $\mathrm{P} 2$ ', and $\mathrm{P} 2$ ' is the point on the line perpendicular to S1. P1 and P2 are corresponding points to be matched exactly if two blocks are assembled accurately. This implies that P2 needs to be moved to the direction toward P2'. As shown in Fig. 2, declination is measured to direct the movement of ship blocks in order to reduce the distance between corresponding points.

In order to measure positional declination efficiently we use a target board, and a CCD camera which captures the image of a target board. A target board has two circles with different sizes and colors, one is enclosed with the other as shown in Fig. 3.

If the center of a target is on the center of a camera view it is regarded that there is no positional declination. So the deviation of two center points is interpreted as the amount of positional declination. If the relative positions of two center points were analyzed, the direction and the distance that a block needs to be moved can be deduced.

Fig. 4 shows some cases that a block has to be moved for right positioning.

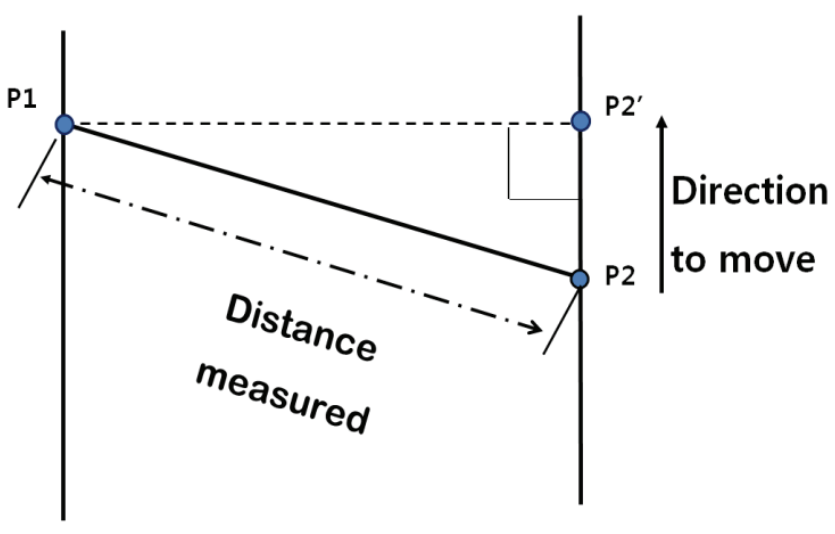

Fig. 2 Concept of a positional declination.

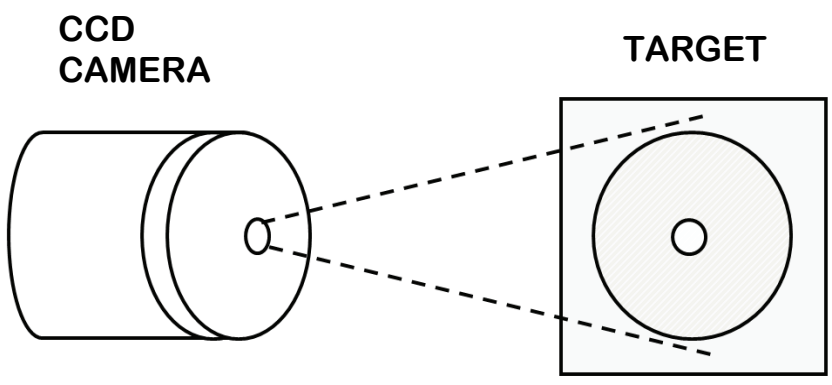

Fig. 3 Devices used to detect positional declination.

\section{Camera's view}

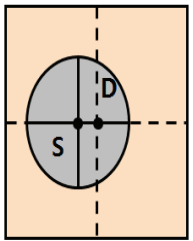

a) to the right

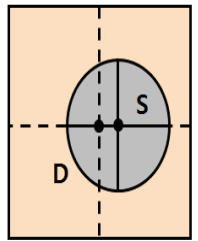

b) to the left

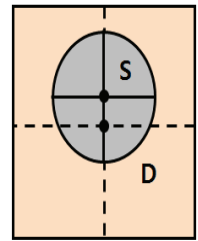

c) to the bottom

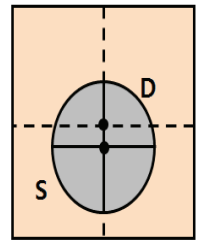

d) To the top
Fig. 4 Some cases of positional declination.

In order to get the center position of a circle, digital image processing on the captured image for a target board is required. After capturing the target board image the circles need to be extracted. In order to do this, a color image has to be transformed into a gray one. By successive process of binarization and labeling, the center point is obtained from the identified circle. With the center point we can calculate position declination as described before. In this process outer circle is used when two blocks are a bit far from each other. By contrast, the inner circle is used if they are very close to each other. Figure5 shows the required processes for estimation of position declination. 


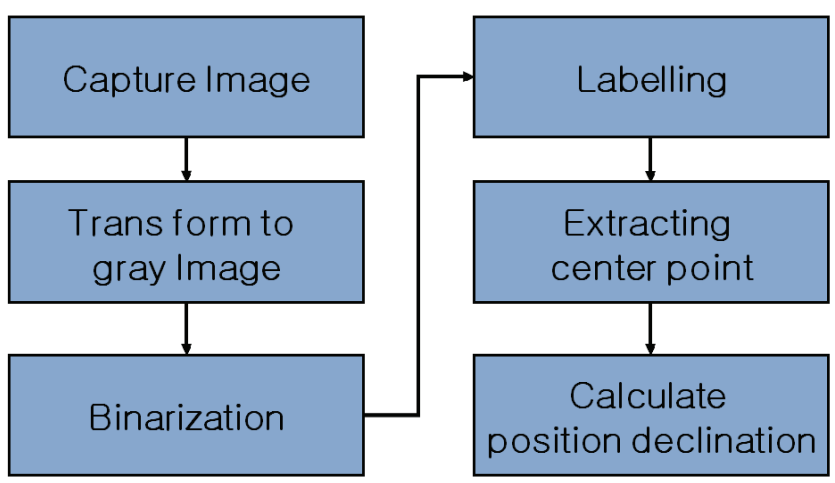

Fig. 5 Processes for calculating position declination.

\section{Data gathering}

Distance-measuring data collected from each sensor are delivered to the monitoring server via a sensor network composed by the sensor nodes. Deploying 'push' approach, each sensor node sends its measured data autonomously to the communication channel by its predetermined rule without considering the status of a monitoring server. A Naive Push (NP) approach is just to push data at every fixed time slot, for example, in every second. As this approach transmits data from a sensor continuously, the volume of transmitted data will be so big. As sensor nodes are operated by battery power, applying more intelligent methods to reduce the data volume will be helpful for more robust and efficient operation.

The utility of the measured distance data may changes according to the distance between two ship blocks. When two ship blocks is about $10 \mathrm{~m}$ apart during block assembly process, sensors need not report the measured distance data in every 1 second because they could not be moved to a very close position in only 1 second. This means that the time interval to acquire distance data might be much larger than 1 second. However if two approaching blocks are only $10 \mathrm{~cm}$ apart, data acquisition needs to be done more frequently so that the distance observer can decide appropriate actions quickly enough to avoid any collision due to late data acquisition. This situation leads to another approach; Adaptive Push(AP) approach to determine the time interval of data delivery based on the distance between two blocks.

In AP approach, a sensor node, $\boldsymbol{i}$, needs to know 3 types of values; measured distance between the ship block $\boldsymbol{m}$ and n, Distance $(\boldsymbol{m}, \boldsymbol{n})$, the threshold value of the distance, C and the time interval, $\boldsymbol{T}$. With this information, a sensor node report its measured distance data, Distance $(\boldsymbol{m}, \boldsymbol{n})$ at every $\boldsymbol{T}$ when the corresponding two ship blocks, $\boldsymbol{m}$ and $\boldsymbol{n}$, are closer than $\boldsymbol{C}$. The AP approach can be implemented in two different ways. The source-based AP (APsrc) approach uses each sensor node to determine the reporting time interval. Each sensor knows when to report its data by referring its local rule used to determine it. A simple example of the specified rule in each sensor node would be as follows;
RULE (i,1): Report Distance $(m, n)$ in every $\mathrm{T}$ seconds when Distance $(m, n)<=\mathrm{C} 1$

RULE (i,2): Report Distance $(m, n)$ in every $\mathrm{P}(\mathrm{P}<\mathrm{T})$ seconds when Distance $(m, n)<=\mathrm{C} 2$ where RULE $(i, j)$ means $\mathrm{j}$-th rule to determine report time at the sensor node i using Distance $(m, n)$.

On the contrary, the sink-based AP (APsink) approach uses a monitoring server to inform reporting time interval to each sensor node. The monitoring server keeps global rules to determine reporting time, and uses monitoring results collected from each sensor node.

When a measured distance data between two blocks falls on each rule, the monitoring server sends the required reporting time interval specified in the rule to the corresponding sensor node.

Table 1 summarizes the characteristics of the proposed approaches described above.

Table 1 Comparison of the data gathering approaches.

\begin{tabular}{|c|c|c|}
\hline & source-based & sink-based \\
\hline $\begin{array}{l}\text { naive } \\
\text { push(NP) }\end{array}$ & $\begin{array}{l}\text { - simple } \\
\text { - needs one-way } \\
\text { communication } \\
\text { - fixed data report rate }\end{array}$ & $\mathrm{N} / \mathrm{A}$ \\
\hline $\begin{array}{l}\text { adaptive } \\
\text { push(AP) }\end{array}$ & $\begin{array}{l}\text { - variable report rate } \\
\text { - needs one-way } \\
\text { communication } \\
\text { - sensor node autonomy } \\
\text { - less data report than NP }\end{array}$ & $\begin{array}{l}\text { - variable report } \\
\text { rate } \\
\text { - needs two-way } \\
\text { communication } \\
\text { - support central } \\
\text { control } \\
\text { - less data report } \\
\text { than NP }\end{array}$ \\
\hline
\end{tabular}

\section{Interpretation of silent data}

The Adaptive-Push approach does not send the measured data from the sensor nodes that are not in the reporting condition on the assumption that the utility of current data is extremely low. When we assemble two ship blocks using sensor monitoring, several pairs of the sensors are attached to each ship blocks at the beginning; then a ship block is moved to the other ship block; sensors continuously measure the distance data; and each sensor node checks reporting condition. This means that some sensors, which fall under the reporting condition, will report its data at some point of time, while others not. We call "silent data" that would have been reported but actually have not been reported based on the the adaptive push rule. We are currently interpreting a silent data of a sensor node as having the same value of the lastly received data from the same sensor node. 


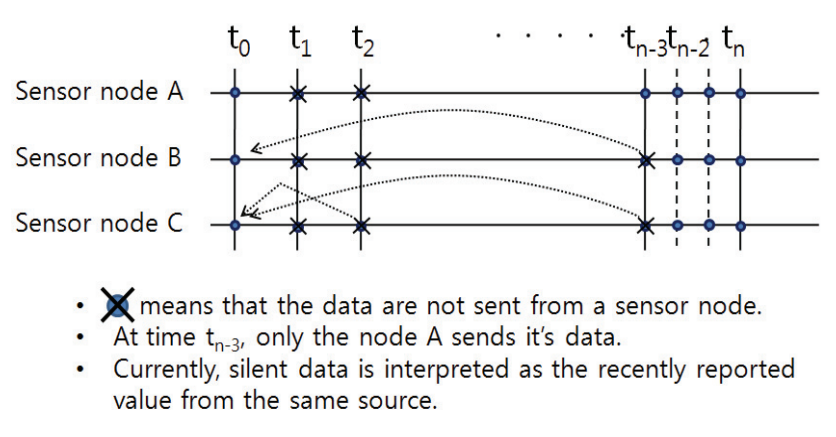

Fig. 6 Interpreting silent data.

\section{PROTOTYPE IMPLEMENTATION OF A SYSTEM}

A prototype system for monitoring of ship block assembly is implemented based on the concepts described before. The system consists of two major system components; the data gathering subsystem which collects distance and position data from the sensors, and the monitoring subsystem which receives the gathered data and supports an observer's decision by analysis of data and display of the block assembly status in real-time. Fig. 7 shows the architecture of the implemented system.

Distance data measuring system uses a reflective type ultrasonic sensor whose measurable range is between $3 \mathrm{~cm}$ and $2 \mathrm{~m}$. For the near distance measuring, linear position transducer with detectable range of $0 \sim 20$ is used. Although the two types of sensors are acquiring their data regardless of the distance between the blocks, which data regarded as valid are determined by the threshold distance predefined in the system. The distance, $20 \mathrm{~cm}$, is adopted as threshold distance to switch the valid source of data, the ultrasonic device will be used to collect distance when the two blocks are apart farther than $20 \mathrm{~cm}$. Otherwise the data from the linear position transducer will be exploited. As a device to capture positional declination we are using an image board which incorporates an ARM processor, a CMOS 1.3Mbps camera and a wireless LAN of $802.11 g$.

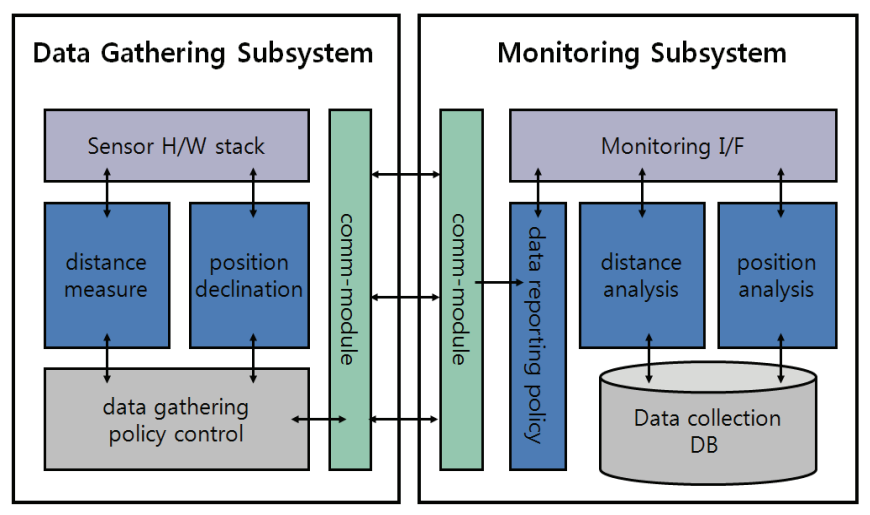

Fig. 7 Architecture of the prototype monitoring system.

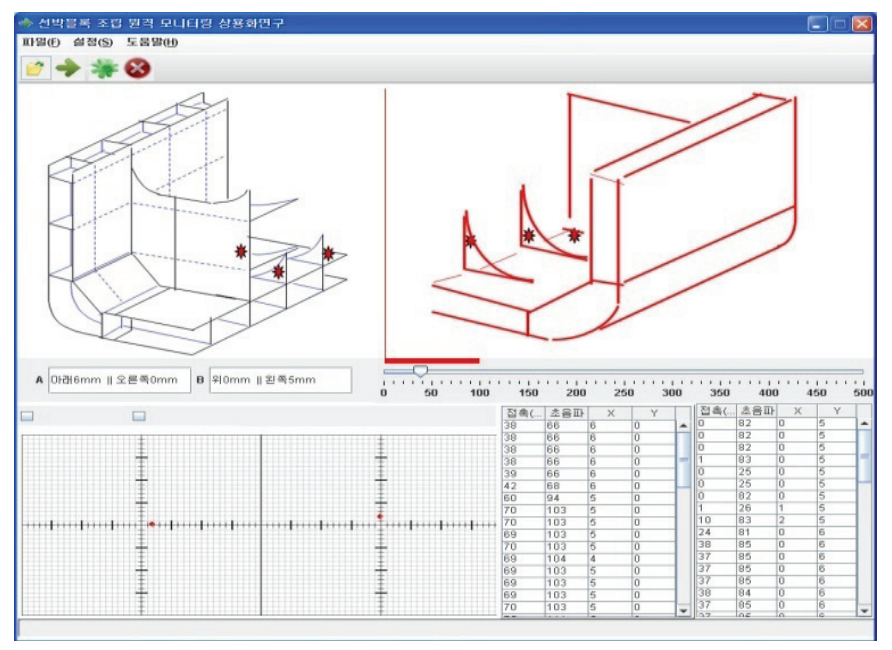

Fig. 8 An example of monitoring interface.

For the monitoring subsystem, a JAVA-based monitoring server has been implemented. After receiving acquired data from the data gathering component, it interprets the data and recommends the required block movement. It also displays current ship block assembly status from the analyzed data. Figure8 shows an example of the interface for ship block assembly monitoring.

\section{CONCLUSIONS}

This paper presents a ship block assembly monitoring system which can improve the productivity in pre-erection or erection stage by applying sensor technology. The most important design issue is to make the proposed approach realistic and feasible.

Unlike other sensor-based applications, ship block assembly applications need high level of precision for measuring distance. In order to solve this problem we have introduced a two-level distance measuring approach which switches distance measuring method based on a threshold distance. Also, digital image processing technology is introduced to measure the positional declination required for right positioning of moving ship blocks.

Several data gathering policies and analyzed pros and cons of the approaches are developed, and it is believed that the source-based adaptive push approach considering "silent data" is effective on reducing message traffics required for data reporting. The developed system is expected to put to practical use shortly.

\section{ACKNOWLEDGEMENTS}

This work was supported by Regional Innovation Center for Southwestern Mid-size Shipbuilding Industry Project in the year of 2010, Mokpo National University. 


\section{REFERENCES}

Bahl, P. and Padmanabhan, V.N., RADAR: 2000. An InBuilding RF-based User Location and Tracking System. Proc. IEEE INFOCOM, pp.775-784.

Fox, D. Burgard, W. Dellaert, F. and Thrun, S., 1999. Monte Carlo Localization: Efficient Position Estimation for Mobile Robots. Proc. of AAAI, pp.343-349.

Gutmann, J.-S. Burgard, W. Fox, D. and Konolige, K., 1998.An Experimental Comparison of Localization Method. Proceedings of Intelligent Robots and Systems, pp.736-743.

Han, K. ed., 2006. A Study on Enhancing Precision for Mid and Small size Ship Block Assembly. Final Report of Regional Industry Promotion \& Development Research, Ministry of Commerce, Industry and Energy.

Hightower, J. and Borriello, G., 2001a. A Survey and Taxonomy of Location Systems for Ubiquitous Computing. Technical Report UW-CSE-01-08-03, University of Washington.

Hightower, J. and Borriello, G., 2001b. Location Sensing Techniques. Technical Report UW-CSE-01-07-01, University of Washington.
Hightower, J. and Borriello, G., 2001c. Location systems for ubiquitous computing, Computer, 34(8), IEEE Computer Society Press.

Kang, Y. Choi, H. Kim, H. Hong, S. and Park, J., 2008. Strategy to Implement RFID in the Marine Equipment Distribution Center. Korea Society of IT Services Journal, 7(1), pp.219-235.

Lee, S, 2009. Sensor-based Remote Monitoring for Ship Block Assembly.Journal of Korea Content, 9(1), pp. 73-80.

Nicolescu, D. and Nath, B., 2001. Ad-Hoc Positioning Systems(APS). Proceedings of IEEE GLOBECOM, pp. 2926-2931.

Priyantha, NB.Chakraborty, A. and Balakrishnan, H., 2000. The Cricket Location-support system. Proc. of the Sixth Annual ACM International Conference on Mobile Computing and Networking (MOBICOM), pp.32-43.

Want, R. Hopper, A. Falcao,V. and Gibbons, J., 1992. The Active Badge Location System. ACM Transactions on Information Systems, 10(1), pp.91-102. 